\title{
Adiponectin is valuable in the diagnosis of acute heart failure with renal insufficiency
}

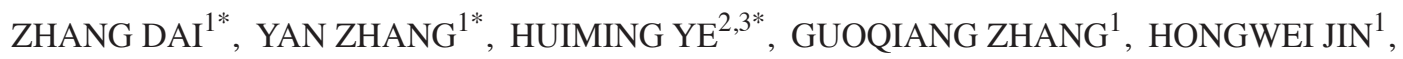 \\ ZIMING CHEN $^{4}$, YIHUI YAO ${ }^{1}$, XUEBING TIAN $^{5}$, JIANFENG ZHOU ${ }^{1}$, PEIHUA LI ${ }^{1}$, \\ XIANMING LIANG ${ }^{1}$, HUABING XIE ${ }^{5}$, SHENGXIANG GE ${ }^{2}$ and ZHONGYING ZHANG ${ }^{1,2,6}$
}

${ }^{1}$ Department of Clinical Laboratory, Zhongshan Hospital Xiamen University, Xiamen, Fujian 361001;

${ }^{2}$ State Key Laboratory of Molecular Vaccine and Molecular Diagnostics, School of Public Health, Xiamen University, Xiamen, Fujian 361002; ${ }^{3}$ Department of Clinical Laboratory, Xiamen Maternal and Child Health Hospital, Teaching Hospital of Medical College Xiamen University, Xiamen, Fujian 361003; ${ }^{4}$ Department of Reagent Research, Xiamen Innovax Biotech Co., Ltd., Xiamen, Fujian 361022; ${ }^{5}$ Department of Clinical Laboratory, Xiamen Cardiovascular Hospital, Medical College Xiamen University; ${ }^{6}$ Department of Clinical Laboratory, Zhongshan Teaching Hospital, Fujian Medical University, Xiamen, Fujian 361001, P.R. China

Received June 12, 2017; Accepted March 27, 2018

DOI: $10.3892 / \mathrm{etm} .2018 .6511$

\begin{abstract}
Acute heart failure (AHF) is a major public health issue due to its high incidence and poor prognosis; thus, efficient and timely diagnosis is critical for improving the prognosis and lowering the mortality rate. Amino-terminal pro-brain natriuretic peptide (NT-proBNP) is widely used in the diagnosis of AHF; however, its efficacy is controversial in diagnosing AHF with renal insufficiency. There were numerous studies reporting the association of adiponectin (ADPN) and heart diseases. Therefore, the present study aimed to investigate whether ADPN is helpful in identifying AHF with renal insufficiency. A total of 407 participants (218 AHF patients and 189 controls) were enrolled into the current study. The plasma levels of ADPN and NT-proBNP were measured using a sandwich enzyme-linked immunosorbent assay and an electrochemiluminescence immunoassay, respectively. In addition, these levels were compared among the various New York Health Association classes, as well as the ischemic and non-ischemic AHF cases. The correlation between the two biomarkers and the renal function was analyzed by Spearman's correlation, while the diagnostic efficiency of ADPN and NT-proBNP was evaluated in AHF patients with and without
\end{abstract}

Correspondence to: Dr Zhongying Zhang, Department of Clinical Laboratory, Zhongshan Hospital Xiamen University, 201 Siming District South Lake Road, Xiamen, Fujian 361001, P.R. China

E-mail: zhongyingzhang_01@126.com

"Contributed equally

Key words: acute heart failure, amino-terminal pro-brain natriuretic peptide, adipocyte-secreted protein, adiponectin, glomerular filtration rate renal insufficiency. The results revealed that NT-proBNP exhibited a higher diagnostic efficiency as compared with ADPN in patients without renal insufficiency [area under the receiver operating characteristic curve (AUC), 0.905 vs. 0.775]. By contrast, the ADPN presented a better diagnostic value in comparison with NT-proBNP in AHF with renal insufficiency (AUC, 0.872 vs. 0.828). Therefore, a combination of these two biomarkers may provide an excellent efficacy in the diagnosis of AHF with renal insufficiency (AUC, 0.904; sensitivity, 71.2\%; specificity, 98.3\%). In conclusion, ADPN is a valuable biomarker for diagnosing AHF, particularly in patients with impaired renal function.

\section{Introduction}

Acute heart failure (AHF) is a major public health issue due to its high incidence and poor prognosis, while the number of hospitalizations for AHF continues to increase due to the aging population (1-4). Efficient and timely diagnosis is critical for improving the prognosis, as well as reducing the mortality rate, length of hospital stay and treatment costs $(5,6)$. Compared with other detection methods for diagnosing AHF, plasma markers have certain advantages, including the simple and easy detection. Amino-terminal pro-brain natriuretic peptide (NT-proBNP) is one of the most commonly used markers in AHF diagnosis (7-9). However, renal dysfunction is a common comorbidity in AHF patients (10). In addition, patients with chronic kidney disease (CKD) present increased risks of accelerated atherosclerosis, nonfatal myocardial infarction, congestive heart failure $(\mathrm{CHF})$, atrial and ventricular arrhythmias, and cardiac death (11). Although NT-proBNP is considered as a marker in AHF diagnosis, the diagnostic value of NT-proBNP in patients with renal insufficiency is still debated (12-15). Therefore, a more effective plasma marker is required for the diagnosis of AHF patients with renal insufficiency. 
A multimarker strategy may help diagnose and determine the prognosis of patients with $\mathrm{AHF}(16)$, particularly in patients with AHF and mild to moderate renal impairment, while multimarker approach based on a panel of serially evaluated biomarkers may provide the greatest prognostic improvement. A previous study demonstrated that seven circulating biomarkers, including NT-proBNP, high sensitivity cardiac troponin T, soluble ST2, growth differentiation factor 15 , cystatin-C, galectin-3 and high-sensitivity $\mathrm{C}$-reactive protein (CRP), measured at baseline and on days 2, 5, 14 and 60 in 1,161 patients provided an improved quick diagnosis (17).

The adipocyte-secreted protein adiponectin (ADPN) is a 247 amino acid peptide, predominantly secreted by adipocytes $(18,19)$. Plasma ADPN is typically high in cats, which are overweight and functions by regulating the level of leptin (20). ADPN is considered as a useful biomarker in metabolic diseases, such as diabetes, high-fat-associated disease and dementia (21-23). In addition, it has been observed that ADPN exerts several protective functions in the peripheral tissues, including insulin sensitizing, anti-inflammatory and anti-oxidative effects, which may benefit various neurodegenerative diseases, such as Alzheimer's disease (24). The role of ADPN in heart diseases has been investigated in numerous studies. For instance, a cross-sectional study revealed that a low ADPN level was associated with diastolic dysfunction in women (25). Another study demonstrated that natriuretic peptides, which are promising candidates for the treatment of CHF, may have a beneficial effect on cardiomyocytes in patients through enhancing the ADPN production by human adipocytes in vitro and in patients with $\mathrm{CHF}$ (26). Furthermore, the circulating ADPN concentration increased in patients with chronic heart failure (HF), and thus it is regarded as a useful novel biomarker in $\mathrm{CHF}$ and $\operatorname{AHF}(27,28)$. A previous study suggested that when the ADPN level is used in conjunction with NT-proBNP in chronic HF, the prognostic value may be improved when compared with the use of each biomarker alone (29). Another study also identified ADPN as a robust biomarker and appropriate therapeutic targets in HF (30).

These aforementioned studies prompt the hypothesis that ADPN may be a useful biomarker in diagnosing AHF patients. However, there are a limited number of studies on whether the circulating level of ADPN was affected by glomerular filtration rate (GFR) and, thus, whether it has superiority in diagnosing AHF patients with renal insufficiency. The aim of the present study was to examine the value of ADPN in the diagnosis of these patients. In total, 407 participants were enrolled into the current study, including 218 participants diagnosed with AHF and 189 participants serving as the control group. ADPN was measured in the participants using an in-house sandwich enzyme-linked immunosorbent assay (ELISA) and NT-proBNP was measured by electrochemiluminescence immunoassay. The levels of circulating ADPN and NT-proBNP in the patients of AHF were also compared between different New York Health Association (NYHA) classes, as well as ischemic and non-ischemic AHF. The correlation between the renal function and the two biomarkers of all participants were compared by Spearman's correlation. Finally, the diagnostic efficiency of ADPN and NT-proBNP was evaluated in patients with and without renal insufficiency.

\section{Patients and methods}

Clinical cohort. The present study enrolled 407 patients with suspected cardiac-associated dyspnea who were admitted to the Emergency Department of the Xiamen Cardiovascular Hospital (Xiamen, China) between April 2015 and June 2015. Among them, 218 patients (53\%) were diagnosed with AHF based on the 'European Society of Cardiology guidelines' (31) and classified according to the NYHA system, as follows: Class I, the patient suffers from heart disease, however, ordinary activities are not restricted by cardiac function; Class II, mild restrictions on the physical activity of patients with heart disease and no symptoms at rest, although patients may experience fatigue, dyspnea or angina following physical activity; Class III, severe restrictions on the physical activity of patients with heart disease with cardiac failure symptoms; and Class IV, patient is unable to participate in any physical activities. In light of the guidelines from the European Society of Cardiology guidelines, the following levels of NT-proBNP were used for excluding AHF patients: $450 \mathrm{pg} / \mathrm{ml}$ in patients aged $<50$ years; $900 \mathrm{pg} / \mathrm{ml}$ between the ages of 50 and 75 years; and $1,800 \mathrm{pg} / \mathrm{ml}$ in patients aged $>75$ years (31). Furthermore, cardiovascular magnetic resonance was conducted to determine whether ischemic AHF was present. The remaining 189 participants presented a normal cardiac function and served as the controls, including patients with bronchial asthma and other respiratory system diseases. Patients receiving dialysis within the three months prior to admission were excluded from the current study since ADPN and NT-proBNP levels are affected by dialysis. The study was approved by the Ethics Committee of Zhongshan Hospital, Xiamen University (Xiamen, China), and was conducted according to the Declaration of Helsinki (2008). Written informed consent was obtained from all participants.

Laboratory measurements. The levels of NT-proBNP and the renal function were detected immediately upon presentation to the Emergency Department. Plasma was collected in an EDTA-K3 anticoagulation tube and centrifuged at 1,006 $\mathrm{x} \mathrm{g}$ at $4^{\circ} \mathrm{C}$ for $15 \mathrm{~min}$. NT-proBNP was detected using the Elecsy proBNP II electrochemiluminescence immunoassay (Roche Diagnostics, Basel, Switzerland) and the Cobas E601 analyzer (Roche Diagnostics). The residual plasma samples were preserved at $-80^{\circ} \mathrm{C}$ for ADPN level detection.

Subsequently, the plasma ADPN levels were detected using a sandwich ELISA kit (Xiamen Innovax Biotech Co., Ltd., Xiamen, China). The serum samples were diluted by 100 times during the ELISA procedure, while the total ADPN level was the sum of several polymers, including trimers, hexamers and high polymers.

Renal function tests, including creatinine and GFR, were performed with an auto-biochemistry analyzer (Cobas C701; Roche Diagnostics). The GFR was estimated using the Modification of Diet in Renal Disease equation as previously described (32). A GFR value of $<60 \mathrm{ml} / \mathrm{min} / 1.73 \mathrm{~m}^{2}$ indicated renal insufficiency, while a GFR value of $\geq 60 \mathrm{ml} / \mathrm{min} / 1.73 \mathrm{~m}^{2}$ was considered to indicate normal renal function.

Statistical analysis. The SPSS version 19.0 statistical software package (IBM Corp., Armonk, NY, USA) was used to analyze 
the data. The Skewness-Kurtosis test was used to determine the distribution of data, and the non-parametric Kruskal-Wallis test was performed if the data were not distributed normally and the values were reported as the median (25th quantile and 75 th quantile). Enumeration data were compared using a $\chi^{2}$ test. The Nemenyi post-hoc test was used to compare between multiple sets of data. Spearman's correlation co-efficient was calculated for the correlation of renal function with the ADPN and NT-proBNP levels. Receiver operating characteristic (ROC) curve analyses were performed and the area under the ROC curve (AUC) was calculated to evaluate the diagnostic accuracy of ADPN and NT-proBNP. Data were analyzed separately for participants with normal and impaired renal functions, and subsequently compared. The Z-test was also performed to determine the statistical difference of the ROC curve. A two-sided P-value of $<0.05$ was considered to indicate a statistically significant difference.

\section{Results}

Clinical characteristics of the participants. A total of 407 participants were enrolled into the present study, including 262 males and 145 females, among them 218 participants were accurately diagnosed with AHF. The clinical characteristics of all participants are listed in Table I. In the AHF group, the plasma creatinine levels of the 218 participants were $0.87(0.71,1.13) \mathrm{mg} / \mathrm{dl}$, while the estimated GFR was $86.26(60.20,106.98) \mathrm{ml} / \mathrm{min} / 1.73 \mathrm{~m}^{2}$. In the control group, the plasma creatinine levels of the 189 participants were 0.97 $(0.80,1.34) \mathrm{mg} / \mathrm{dl}$ and the estimated GFR was 75.04 (54.18, $95.08) \mathrm{ml} / \mathrm{min} / 1.73 \mathrm{~m}^{2}$. The reference values for creatinine and GFR were $0.60-1.09 \mathrm{mg} / \mathrm{dl}$ and $90-120 \mathrm{ml} / \mathrm{min} / 1.73 \mathrm{~m}^{2}$, respectively. Renal insufficiency was detected in 111 participants, among which 52 were diagnosed with AHF. Renal insufficiency was detected in 59 individuals within the control group. In addition, the plasma ADPN level was 11.05 $(6.51,16.28) \mu \mathrm{g} / \mathrm{ml}$ and the NT-proBNP was $2,158(1,032$, $4,930) \mathrm{pg} / \mathrm{ml}$ in the AHF group, whereas the ADPN level was $5.56(3.43,7.39) \mu \mathrm{g} / \mathrm{ml}$ and the NT-proBNP was $220(56,690)$ $\mathrm{pg} / \mathrm{ml}$ in the control group.

ADPN levels are significantly associated with the NYHA class, and may assist in distinguishing non-ischemic from ischemic $A H F$. The results revealed that ADPN and NT-proBNP levels were significantly higher in participants with an advanced NYHA class (Table II; Fig. 1A and B). Statistically significant differences were detected in the ADPN levels between the control group and the NYHA class I $(\mathrm{P}=0.04)$; however, there was no significant difference in the NT-proBNP level between these groups $(\mathrm{P}=0.26)$. In terms of the plasma ADPN level, significant differences were detected between class I and II $(\mathrm{P}=0.12)$, class II and III $(\mathrm{P}=0.00)$, and class III and IV $(\mathrm{P}=0.04)$. Regarding the plasma NT-proBNP levels, marked differences were observed between class I and II $(\mathrm{P}=0.02)$ and class III and IV $(\mathrm{P}=0.02)$.

Participants with AHF were further divided into the ischemic and non-ischemic HF groups based on the disease pathogenesis of AHF. The non-ischemic group included cases of valvular heart disease, hypertension, dilated cardiomyopathy, hypertrophic cardiomyopathy and hypertensive heart disease. The ischemic group included coronary artery disease and atherosclerosis heart disease cases. As shown in Table III and Fig. 1C and D, the ADPN levels in the ischemic group were significantly reduced when compared with those in the non-ischemic group $(\mathrm{P}=0.02)$. However, there was no statistically significant difference detected in the NT-proBNP levels between the two groups $(\mathrm{P}=0.81)$. The combination of these two indicators may help clinicians to establish a timely and accurate diagnosis of AHF, and to subsequently select the appropriate treatment.

Circulating ADPN levels are affected by renal function to a lesser extent as compared with NT-proBNP levels. The GFR value of the participants was determined to examine the renal function (renal insufficiency: GFR $\geq 60$ ). The levels of NT-proBNP $(\mathrm{pg} / \mathrm{ml})$ in both the AHF and control (normal cardiac function) patients were significantly higher in individuals with renal insufficiency as compared with those with normal renal function [AHF renal insufficiency 4,697.0 $(1,878.5,10,744.5)$ vs. normal renal function $1,892.0(892.5$, $3,870.0)$ and Control renal insufficiency 656.0 (316.8, 2,172.5) vs. normal renal function $104.0(37.0,331.0)$, respectively; both $\mathrm{P}<0.01$; Fig. 2A and B]. Similarly, the levels of ADPN $(\mu \mathrm{g} / \mathrm{ml})$ in AHF patients were also significantly affected by the renal function, since the ADPN level in AHF patients with renal insufficiency was significantly higher compared with those with normal renal function [AHF renal insufficiency 12.9 $(8.6,16.5)$ vs. normal renal function 10.6 (6.2, 15.7); $\mathrm{P}=0.04$; Fig. 2C]. By contrast, in control patients (normal cardiac function), there was no evident difference in the ADPN level between the normal and abnormal renal function groups [renal insufficiency $5.8(3.7,7.5)$ vs. normal renal function 5.3 (3.3, 7.2); $\mathrm{P}=0.62$; Fig. 2D).

The correlation between AHF serum biomarkers (NT-proBNP and ADPN) and renal function were then further investigated. In the present study, the levels of creatinine and GFR were considered to reflect the renal function. As shown in Fig. 3A and B, the NT-proBNP levels were significantly correlated with the renal function. The NT-proBNP levels were positively correlated with creatinine and negatively correlated with GFR in both the AHF and control groups. The correlation coefficients in the control and AHF groups were $0.386(\mathrm{P}<0.01)$ and $0.343(\mathrm{P}<0.01)$ for creatinine, respectively, and $-0.488(\mathrm{P}<0.01)$ and $-0.412(\mathrm{P}<0.01)$ for GFR, respectively. By contrast, as shown in Fig. $3 \mathrm{C}$ and D, ADPN was not significantly correlated with creatinine and GFR. In the control and AHF groups, the correlation coefficients for creatinine were observed to be $0.124(\mathrm{P}=0.12)$ and $0.073(\mathrm{P}=0.29)$, respectively, while for GFR, the coefficients were $0.008(\mathrm{P}=0.21)$ and $-0.177(\mathrm{P}=0.16)$, respectively. These findings indicated that the ADPN level was affected to a lesser extent by impaired renal function as compared with the NT-proBNP level, which may be helpful in diagnosing AHF patients with impaired renal function.

Diagnostic value of ADPN and NT-proBNP for AHF in normal renal function and impaired renal function patients. To determine whether the plasma levels of ADPN and NT-proBNP had diagnostic values in AHF, the ROC curve analysis was applied to examine their diagnostic 
Table I. Clinical characteristics of 407 participants.

\begin{tabular}{|c|c|c|c|}
\hline Characteristics & $\mathrm{AHF}$ & Control & P-value \\
\hline No. of cases $(n)$ & 218 & 189 & 0.80 \\
\hline Diabetes mellitus (n) & 123 & 109 & 0.31 \\
\hline Smoking (n) & 58 & 42 & \\
\hline Age $(\text { years })^{\mathrm{a}}$ & $63(51,74)$ & $63(55,71)$ & 0.14 \\
\hline Weight $(\mathrm{kg})^{\mathrm{a}}$ & $62(53,72)$ & $63(57,76)$ & 0.21 \\
\hline Height $(\mathrm{m})^{\mathrm{a}}$ & $1.65(1.61,1.73)$ & $1.67(1.63,1.74)$ & 0.18 \\
\hline $\operatorname{BMI}\left(\mathrm{kg} / \mathrm{m}^{2}\right)^{\mathrm{a}}$ & $23.34(21.39,26.76)$ & $22.78(20.97,25.47)$ & 0.27 \\
\hline NT-proBNP $(\mathrm{pg} / \mathrm{ml})^{\mathrm{a}}$ & $2,158(1,032,4,930)$ & $220(56,690)$ & $<0.001$ \\
\hline $\operatorname{ADPN}(\mu \mathrm{g} / \mathrm{ml})^{\mathrm{a}}$ & $11.05(6.51,16.28)$ & $5.56(3.43,7.39)$ & $<0.001$ \\
\hline \multicolumn{4}{|l|}{ Renal function ${ }^{\mathrm{a}}$} \\
\hline CREA (mg/dl) & $0.87(0.71,1.13)$ & $0.97(0.80,1.34)$ & \\
\hline $\operatorname{GFR}\left(\mathrm{ml} / \mathrm{min} / 1.73 \mathrm{~m}^{2}\right)$ & $86.26(60.2,106.98)$ & $75.04(54.18,95.08)$ & \\
\hline Renal insufficiency & 52 & 59 & \\
\hline Normal renal function & 166 & 130 & \\
\hline Systolic pressure $(\mathrm{mmHg})^{\mathrm{a}}$ & $124(108,143)$ & $129(114,147)$ & \\
\hline \multicolumn{4}{|l|}{ Pathogenesis (n) } \\
\hline Non-ischemic & 138 & - & \\
\hline Ischemic & 80 & - & \\
\hline \multicolumn{4}{|l|}{ NYHA class (n) } \\
\hline $\mathrm{I}$ & 9 & - & \\
\hline II & 61 & - & \\
\hline III & 91 & - & \\
\hline IV & 57 & - & \\
\hline
\end{tabular}

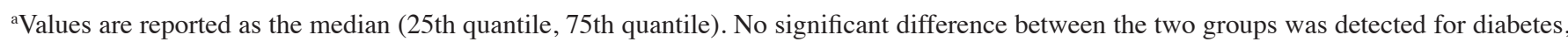
smoking and obesity by the $\chi^{2}$ test. BMI, body mass index; NT-proBNP, amino-terminal pro-brain natriuretic peptide; ADPN, adiponectin; CREA, creatinine; GFR, glomerular filtration rate; NYHA, New York Health Association.

Table II. Levels of ADPN and NT-proBNP in the control group and in heart failure patients with different NYHA cardiac function classifications.

\begin{tabular}{lcc}
\hline Group & NT-proBNP $(\mathrm{pg} / \mathrm{ml})$ & ADPN $(\mu \mathrm{g} / \mathrm{ml})$ \\
\hline Control $(\mathrm{n}=189)$ & $219.5(55.5,690.0)$ & $5.6(3.4,7.4)$ \\
NYHA class & & \\
I (n=9) & $152.0(119.0,664.0)$ & $8.2(5.6,8.6)$ \\
II $(\mathrm{n}=61)$ & $1,367.0(759.0,3706.0)$ & $10.0(5.7,11.2)$ \\
III $(\mathrm{n}=91)$ & $2,158.0(1,105.5,4,152.0)$ & $11.5(6.0,16.6)$ \\
IV $(\mathrm{n}=57)$ & $3,986.0(1,798.0,9,233.0)$ & $14.8(10.1,22.8)$
\end{tabular}

Values are presented as the median (25th quantile, 75th quantile). NT-proBNP, amino-terminal pro-brain natriuretic peptide; ADPN, adiponectin; NYHA, New York Health Association.

efficiency. As shown in Table IV and Fig. 4A, among the patients with a normal renal function $(n=296)$, NT-proBNP presented a significantly better diagnostic efficiency for AHF (AUC $=0.905 ; 95 \% \mathrm{CI}=0.866-0.936$ ) in comparison with that of ADPN (AUC=0.775; 95\% CI=0.723-0.821; $\mathrm{P}<0.001$ ). The optimal cutoff value for NT-proBNP was $296.0 \mathrm{pg} / \mathrm{ml}$, while
Table III. Levels of ADPN and NT-proBNP in the serum of normal, ischemic and non-ischemic groups.

\begin{tabular}{lcc}
\hline Group & ADPN $\mu \mathrm{g} / \mathrm{ml}$ & NT-proBNP pg/ml \\
\hline $\begin{array}{l}\text { Normal } \\
(\mathrm{n}=189)\end{array}$ & $5.6(3.4,7.4)$ & $219.5(55.5,690.0)$ \\
$\begin{array}{l}\text { Ischemic } \\
(\mathrm{n}=80)\end{array}$ & $9.8(6.0,13.2)$ & $2,376.0(935.5,5,896.5)$ \\
$\begin{array}{l}\text { No-ischemic } \\
(\mathrm{n}=138)\end{array}$ & $11.2(6.9,18.7)$ & $1,898.0(1,116.5,3,697.0)$ \\
\hline
\end{tabular}

Values are presented as the median (25th quantile, 75th quantile). NT-proBNP, amino-terminal pro-brain natriuretic peptide; ADPN, adiponectin.

the sensitivity and specificity were 92.1 and $72.3 \%$, respectively. ADPN achieved an optimal diagnostic efficiency at the threshold of $10.6 \mu \mathrm{g} / \mathrm{ml}$, while the sensitivity and specificity were 43.9 and $94.9 \%$, respectively. Compared with NT-proBNP alone, the combination of the two biomarkers did not exhibit a higher diagnostic efficiency $(\mathrm{AUC}=0.920$; 95\% CI, 0.883-0.948; $\mathrm{P}=0.12$ ). 
Table IV. Diagnostic value of ADPN, NT-proBNP and their combination in acute heart failure patients with impaired or normal renal function.

\begin{tabular}{|c|c|c|c|c|c|c|}
\hline \multirow[b]{2}{*}{ Parameter } & \multicolumn{2}{|c|}{ ADPN } & \multicolumn{2}{|c|}{ NT-proBNP } & \multicolumn{2}{|c|}{ Combination } \\
\hline & $\begin{array}{l}\text { Normal renal } \\
\text { function }\end{array}$ & $\begin{array}{c}\text { Renal } \\
\text { insufficiency }\end{array}$ & $\begin{array}{l}\text { Normal renal } \\
\text { function }\end{array}$ & $\begin{array}{c}\text { Renal } \\
\text { insufficiency }\end{array}$ & $\begin{array}{c}\text { Normal } \\
\text { renal function }\end{array}$ & $\begin{array}{c}\text { Renal } \\
\text { insufficiency }^{\mathrm{b}}\end{array}$ \\
\hline Diagnosis efficiency & $77.5 \%$ & $87.2 \%$ & $90.5 \%$ & $82.8 \%$ & $92.0 \%$ & $90.0 \%$ \\
\hline $\mathrm{CI}$ & $72.3-82.1 \%$ & $79.5-89.3 \%$ & $86.6-93.6 \%$ & $74.5-89.3 \%$ & $88.3-94.8 \%$ & $83.3-95.3 \%$ \\
\hline Cut-off & $10.6 \mu \mathrm{g} / \mathrm{ml}$ & $10.6 \mu \mathrm{g} / \mathrm{ml}$ & $296.0 \mathrm{pg} / \mathrm{ml}$ & $1,129.0 \mathrm{pg} / \mathrm{ml}$ & $35.0 \%$ & $75.0 \%$ \\
\hline Sensitivity & $43.9 \%$ & $65.4 \%$ & $93.1 \%$ & $90.4 \%$ & $95.0 \%$ & $71.2 \%$ \\
\hline Specificity & $94.9 \%$ & $91.5 \%$ & $72.3 \%$ & $67.8 \%$ & $75.9 \%$ & $98.3 \%$ \\
\hline PPV & $91.8 \%$ & $87.2 \%$ & $79.6 \%$ & $71.2 \%$ & $92.9 \%$ & $93.8 \%$ \\
\hline NPV & $61.9 \%$ & $75.0 \%$ & $81.8 \%$ & $88.9 \%$ & $82.1 \%$ & $61.1 \%$ \\
\hline
\end{tabular}

${ }^{a}$ Logistic combination $=0.159 \times$ YAPN + 2.712 x Ylg NT-proBNP-8.533; ${ }^{b}$ Logistic combination $=0.327 \times$ YAPN $+1.657 \times$ Ylg NT-proBNP -8.30 ADPN, adiponectin; NT-proBNP, amino-terminal pro-brain natriuretic peptide; PPV Combination $=0.327 \times \mathrm{Y}_{\mathrm{APN}}+1.657 \mathrm{x} \mathrm{Y}_{1 \mathrm{gNT} \text {-proBNP}}-8.30$ NT-proBNP, amino-terminal pro-brain natriuretic peptide; ADPN, Adipocyte-secreted protein Adiponectin.

A

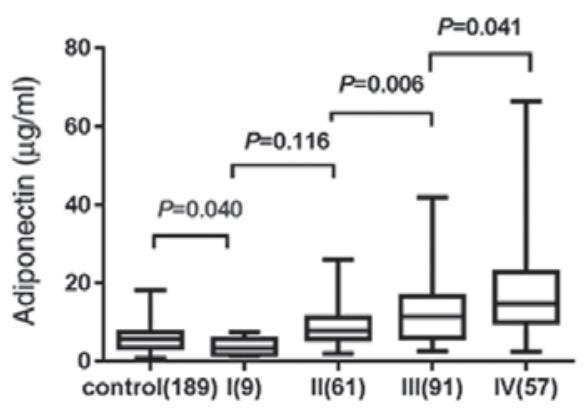

C

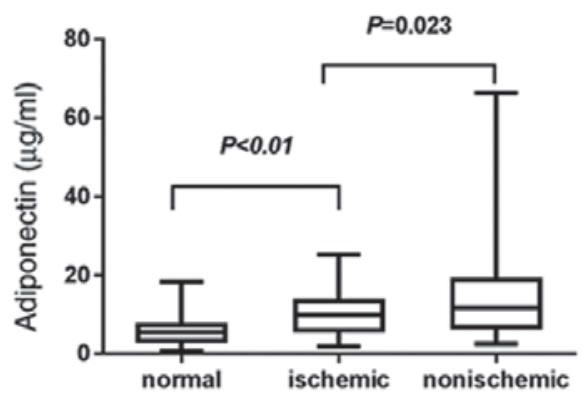

B
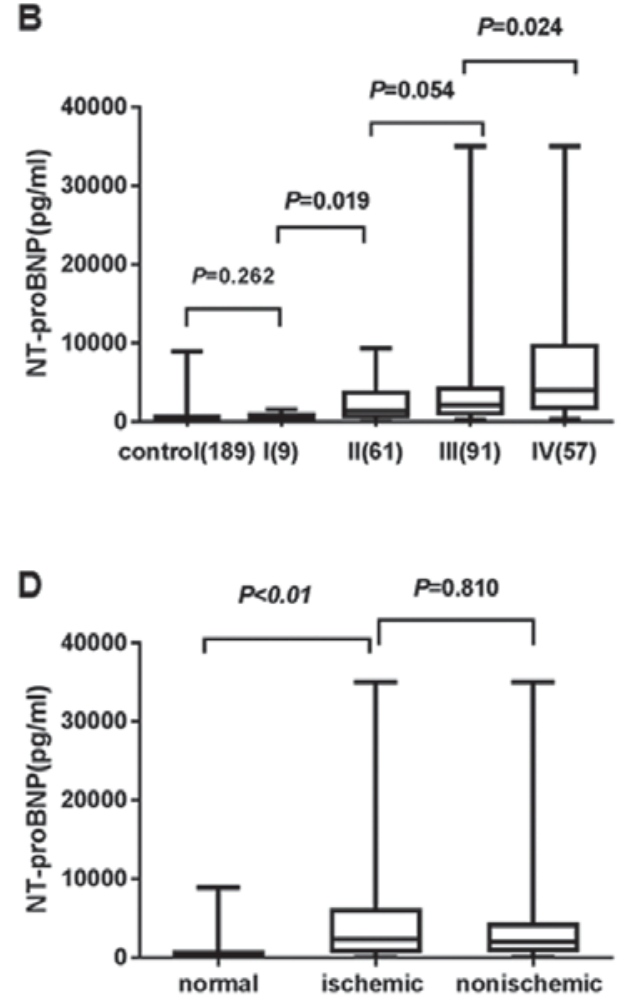

Figure 1. Comparative levels of plasma NT-proBNP and ADPN in different NYHA classes, as well as in ischemic and non-ischemic HF. Plasma (A) ADPN and (B) NT-proBNP levels in the control group and AHF patients divided according to the NYHA class. Plasma (C) ADPN and (D) NT-proBNP levels in patients with normal, ischemic and non-ischemic HF. Differences between normal and ischemic HF was statistically significant for ADPN (P<0.01) and NT-proBNP $(\mathrm{P}<0.01)$, while the difference between ischemic and non-ischemic HF was statistically significant only for ADPN but not NT-proBNP $(\mathrm{P}=0.02$ vs. $\mathrm{P}=0.81$, respectively). AHF, acute heart failure; NT-proBNP, amino-terminal pro-brain natriuretic peptide; ADPN, adiponectin; NYHA, New York Health Association.

As shown in Table IV and Fig. 4B, among patients with an abnormal renal function ( $\mathrm{n}=111)$, ADPN presented a better diagnostic efficiency (AUROC $=0.872 ; 95 \% \mathrm{CI}, 0.795-0.928$ ) in comparison with that of NT-proBNP (AUROC $=0.828$, 95\%CI: 0.745-0.893), although the difference was not statistically significant $(\mathrm{P}=0.34)$. The optimal cutoff value for NT-proBNP in the renal insufficiency patients was
$1,129.0 \mathrm{pg} / \mathrm{ml}$, with sensitivity and specificity of 90.4 and $67.8 \%$, respectively. The optimal threshold for ADPN was also $10.6 \mu \mathrm{g} / \mathrm{ml}$, and the sensitivity and specificity were 65.4 and $91.5 \%$, respectively. Compared with NT-proBNP alone, combination of the two biomarkers significantly increased the diagnostic efficiency (AUC $=0.90 ; 95 \%$ CI, 0.833-0.951; $\mathrm{P}=0.02)$. Compared with ADPN alone, combination of the 
Table V. Comparative diagnostic efficiencies of ADPN and NT-proBNP in participants in different age groups.

\begin{tabular}{lcccccrrr}
\hline & \multicolumn{2}{c}{ ADPN } & & \multicolumn{2}{c}{ NT-proBNP } & & \multicolumn{2}{c}{ Combination } \\
\cline { 2 - 3 } Parameter & $>75$ years & $50-75$ years & & $<50$ years & $>75$ years & & $50-75$ years & $<50$ years \\
\hline AUC (\%) & 78.9 & 75.9 & & 79.7 & 90.6 & 90.8 & 91.3 \\
Cut-off & 10.6 & 10.6 & & 10.6 & $1,497.0$ & & 335.0 & 221.5 \\
Sensitivity (\%) & 67.9 & 44.1 & & 52.6 & 78.6 & & 94.6 & 92.1 \\
Specificity (\%) & 87.5 & 95.1 & & 100 & 93.7 & & 78.8 & 72.7 \\
PPV (\%) & 71.4 & 78.4 & & 95.8 & 73.9 & & 80.7 & 85.3 \\
NPV (\%) & 56.5 & 70.3 & & 58.3 & 71.4 & & 94.0 & 84.2 \\
\hline
\end{tabular}

ADPN, adiponectin; NT-proBNP, amino-terminal pro-brain natriuretic peptide; AUC, area under the curve.
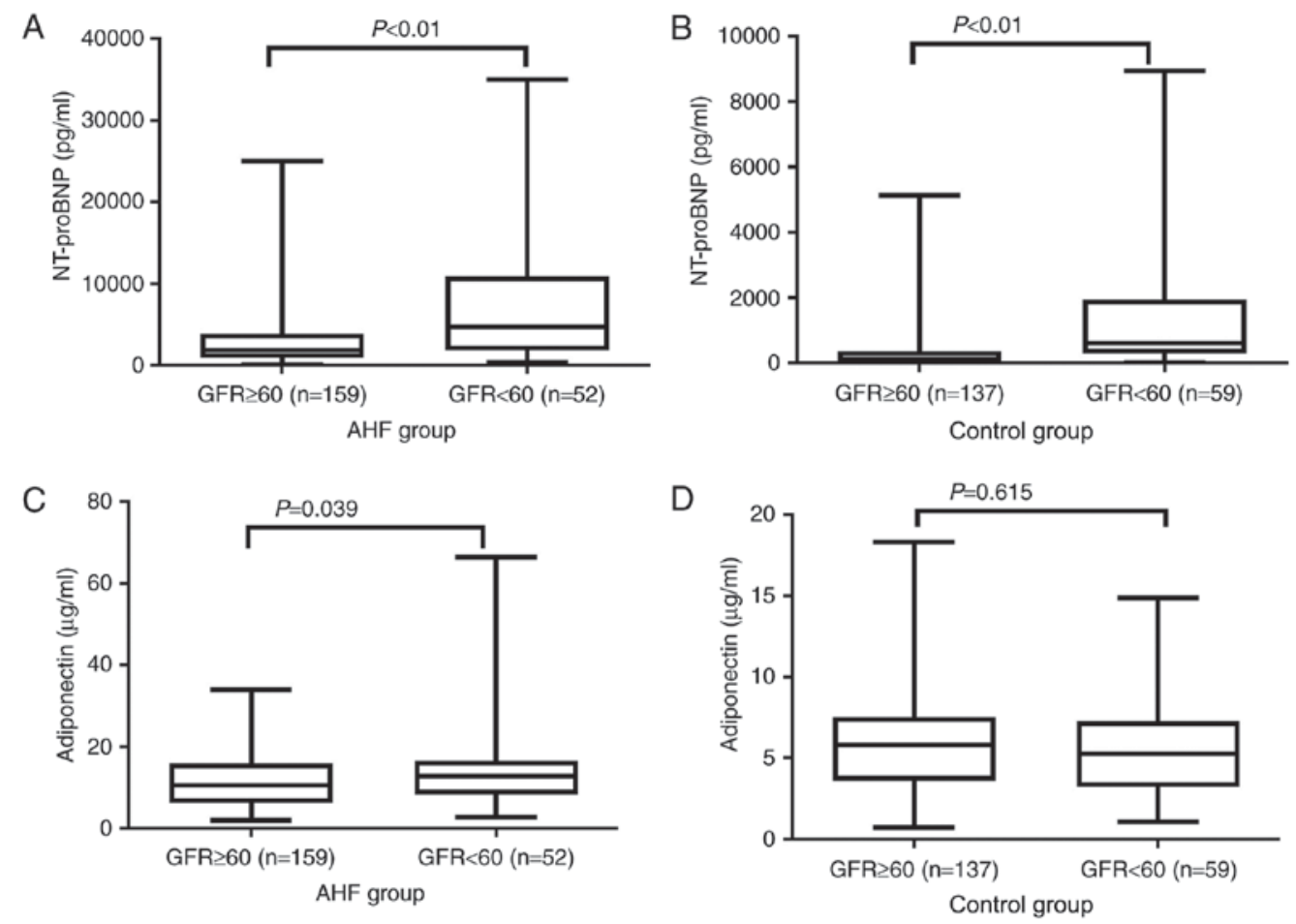

Figure 2. Comparative levels of ADPN or NT-proBNP in the AHF patients and controls, with (GFR $\geq 60)$ and without (GFR $<60)$ renal function insufficiency. NT-proBNP levels in the (A) AHF patients and (B) controls with normal or abnormal renal function are shown. ADPN levels in (C) AFH patients and (D) controls with normal or abnormal renal function are displayed. AHF, acute heart failure; NT-proBNP, amino-terminal pro-brain natriuretic peptide; ADPN, adiponectin; GFR, glomerular filtration rate.

two biomarkers also significantly increased the diagnostic efficiency $(\mathrm{P}=0.04)$. Combined analysis of the two biomarkers demonstrated a higher area under the curve $(\mathrm{AUC}=0.90)$ and specificity (98.3\%), but with lower sensitivity (70.8\%), in diagnosing AHF in patients.

Diagnostic efficiency of ADPN in AHF is less affected by age than NT-proBNP. As shown in Table V, the optimal diagnostic cutoff values of NT-proBNP and ADPN in different age groups were analyzed. Patients with a normal renal function $(n=218)$ were divided into three groups according to their age, namely the groups of $\geq 75,50-75$ and $\leq 50$ years. The plasma NT-proBNP levels were affected by the age of patients. The present study revealed that the optimal cut-off values of NT-proBNP in diagnosing AHF were 221.5, 335.0 and $1,497.0 \mathrm{pg} / \mathrm{ml}$ in these groups, respectively.

The AUC in individuals aged $>75$ years was $78.9 \%$ when ADPN was $10.6 \mu \mathrm{g} / \mathrm{ml}$, and the sensitivity and specificity were 67.9 and $87.5 \%$, respectively (Table V). The positive and negative predictive rates were 71.4 and $56.5 \%$, respectively. Within the group aged 50-75 years, the AUC was $75.9 \%$, the sensitivity and specificity were 44.1 and $95.1 \%$, respectively and the positive and negative predictive rates were 78.4 and $70.3 \%$, respectively. In the group aged $<50$ years, the sensitivity and specificity were 52.6 and $100.0 \%$, respectively and the positive and negative predictive rates were 95.8 and $58.3 \%$ respectively. These results suggest that as a diagnostic marker ADPN was insensitive to age, however it had a lower diagnosis efficiency compared with NT-proBNP. 

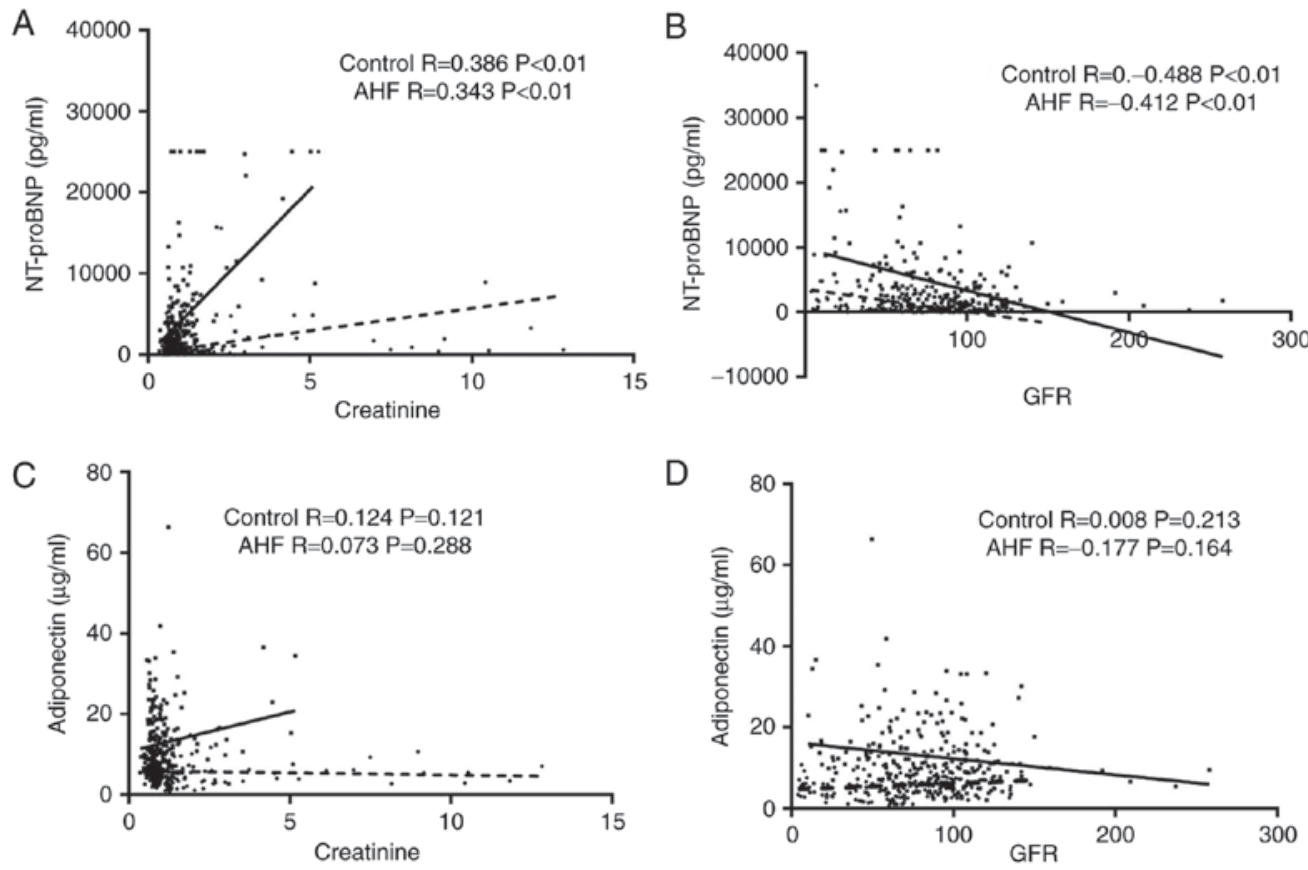

Figure 3. Comparative correlation of ADPN or NT-proBNP with renal function in the control and AHF groups. Renal function was examined according to the creatinine level and GFR. Correlation between NT-proBNP and (A) creatinine or (B) GFR in the control and AHF groups. The correlation coefficients were $0.386(\mathrm{P}<0.01)$ and $0.343(\mathrm{P}<0.01)$ for creatinine, respectively, and $-0.488(\mathrm{P}<0.01)$ and $-0.412(\mathrm{P}<0.01)$ for GFR, respectively. The correlation of ADPN with (C) creatinine or (D) GFR in the control and AHF groups is also displayed. The correlation coefficients were $0.124(\mathrm{P}=0.12)$ and $0.073(\mathrm{P}=0.29)$ for creatinine, respectively, and $0.008(\mathrm{P}=0.21)$ and $-0.177(\mathrm{P}=0.16)$ for $\mathrm{GFR}$, respectively. AHF, acute heart failure; NT-proBNP, amino-terminal pro-brain natriuretic peptide; ADPN, adiponectin; GFR, glomerular filtration rate.

A

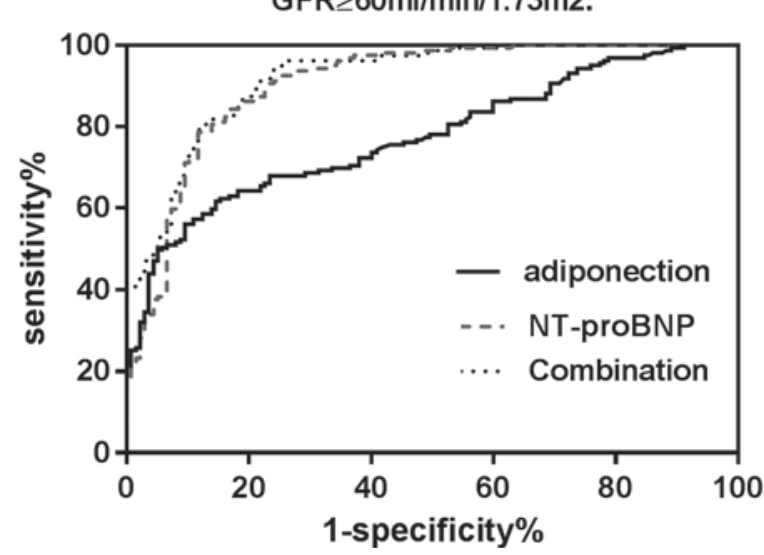

B

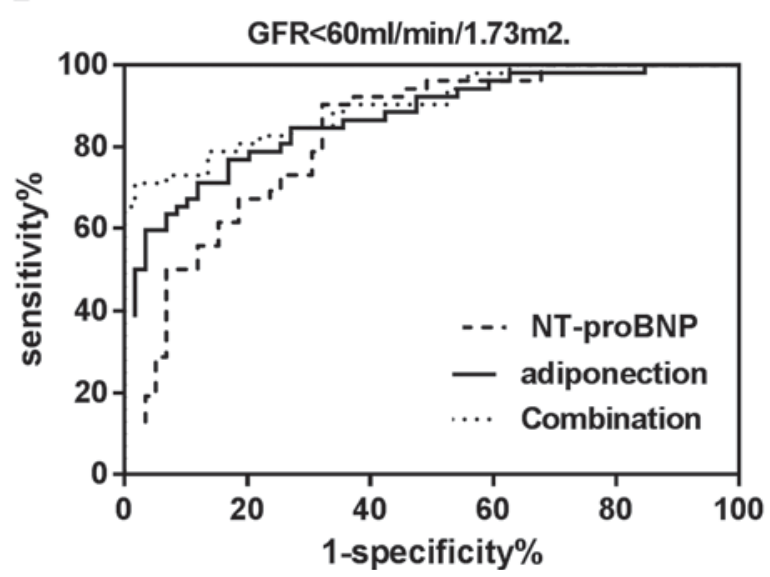

Figure 4. Receiver operating characteristic curve of adiponectin, NT-proBNP and their combination in acute heart failure patients with (A) normal renal function and (B) renal insufficiency. NT-proBNP, amino-terminal pro-brain natriuretic peptide.

\section{Discussion}

AHF is the most common cause of unplanned hospital admissions, and is associated with high mortality rates $(2,33)$, with the cost of managing AHF being a global burden (34). Efficient and timely diagnosis is critical for reducing the mortality rates (35). NT-proBNP has long been used as a routine and rapid diagnostic method for patients with AHF (7-9); however, the use of NT-proBNP in diagnosing AHF in patients with renal insufficiency is controversial, since its level is markedly affected by the renal function (13-15,35). A significant proportion of patients exhibit functional impairment of both the heart and kidneys $(36,37)$. Thus, diagnosing AHF patients with renal insufficiency rapidly and correctly is challenging in clinical practice.

ADPN is a 247 amino acid peptide that is predominantly secreted by adipocytes (18) and is recognized as a useful biomarker in numerous diseases, including fat-associated and heart diseases $(19,29,30,38)$. As an adipokine, ADPN has anti-inflammatory and cardioprotective effects (39). The present study results demonstrated that plasma ADPN levels increased with the increase of the NYHA class of patients (Fig. 1A), which was consistent with the findings of previous studies (28-30). In addition, previous results indicated that ADPN may have an anti-inflammatory and 
anti-atherosclerotic role $(40,41)$. Another study revealed that regular aerobic exercise decreased the potential risk of coronary heart disease by improving the plasma levels of interleukin-6, ADPN, leptin and CRP (42). Furthermore, a cohort study performing long-term follow-up of the glucose tolerance in patients with acute myocardial infarction indicated that elevated levels of ADPN predicted the outcome following acute myocardial infarction (43), while low ADPN levels may, indicate coronary artery disease $(25,44)$. In the present study, the levels of ADPN were found to be lower in ischemic heart disease as compared with those in non-ischemic AHF (Fig. 1C and Table III), which was consistent with the aforementioned results. It was also observed that the level of ADPN in NYHA cardiac function I participants was higher compared with that of the control group, the difference was statistically significant between the two groups $(\mathrm{P}=0.04)$; by contrast, NT-proBNP did not exhibit a marked difference between these two groups.

In the present study, the NT-proBNP levels were observed to be significantly correlated with the renal function and age of participants (Tables IV and V), and the cut-off value for diagnosing AHF evidently varied in the presence of renal insufficiency, which made it difficult for physicians to determine whether the nephropathy was associated with HF. In addition, the cut-off value of ADPN was not altered in the presence of renal insufficiency, which facilitated the diagnosis. The use of ADPN as a marker of AHF exhibited superiority since it was not affected by the age and renal function of the patients (Tables IV and V). The ADPN receptor 2 is mainly distributed in the liver, and only a small amount will be filtered out through the kidneys, indicating that ADPN is less susceptible to the GFR in comparison with NT-proBNP $(45,46)$. In the current study, compared with NT-proBNP, the same cut-off value of ADPN could be utilized to diagnose patients with AHF. Furthermore, the combination of ADPN and NT-proBNP achieved a significantly higher diagnostic value compared with NT-proBNP alone in patients with renal sufficiency. Thus, ADPN may serve as a novel biomarker in the diagnosis of AHF in patients with abnormal renal function.

Through the ROC curve analysis of the diagnostic value of ADPN and NT-proBNP, the results of the present study indicated that ADPN was not associated with the renal function and age. While the sensitivity of NT-proBNP was higher compared with that of ADPN, the specificity of NT-proBNP was lower than that of ADPN. ADPN and NT-proBNP were used to obtain a logistic regression equation, and the predictor of the equation was used to diagnose AHF. The efficiency of the combination of ADPN and NT-proBNP was better in diagnosing AHF patients with renal insufficiency. Although the influence of dialysis on the diagnostic efficiency of these markers was not discussed in the present study, there was no doubt in assessing the diagnostic efficiency of ADPN for AHF patients with renal insufficiency based on the aforementioned results.

Several studies have revealed the association of serum ADPN levels with the degree of renal failure. For instance, the association of ADPN with CKD was demonstrated recently in a case-control study conducted by Lim et al (47) in 450 CKD cases and 920 controls involving Chinese and Indian adults aged 40-80 years. The authors observed that a higher level of serum ADPN was positively associated with CKD independently of traditional risk factors in the examined Asian population (48). In other studies, higher ADPN levels were reported in end-stage renal disease $(49,50)$, whereas the role of ADPN in mildly impaired renal function was inconsistent, with a previous study demonstrating lower levels of ADPN were associated with CKD (51), while others reporting that higher levels were associated with CKD or no significant association was observed as discussed by Lim et al (47).

The present study has certain limitations. Firstly, only two markers were measured for diagnosing AHF. In addition, the sample size of the study and the number of participants with renal insufficiency were small. Furthermore, patients with severe renal impairment were not represented adequately in the current study. Thus, a large-scale trial comparing several different markers of AHF is required in order to further evaluate the diagnostic accuracy of ADPN and NT-proBNP in patients with renal insufficiency. Finally, patients with hemodialysis need to be enrolled in order to further evaluate the diagnostic accuracy of each biomarker.

In conclusion, NT-proBNP demonstrated a higher diagnostic efficiency compared with ADPN in AHF patients without renal insufficiency, while ADPN presented a better diagnostic value. Therefore, a combination of these two biomarkers may provide improved efficacy in the diagnosis of AHF with renal insufficiency. In addition, the present study observed that ADPN was less affected by the renal function and age of patients, and can be used for diagnosing AHF, particularly in patients with impaired renal function.

\section{Acknowledgements}

Not applicable.

\section{Funding}

The present study was supported by the Major Project of Department of Science and Technology of Fujian Province (grant no. 2011D018), the Scientific and Technological Project of Xiamen City (grant no. 3502Z20130006), and the Youth Foundation of Fujian Province Health Department (grant no. 2012-2-83).

\section{Availability of data and materials}

The datasets used during the present study are available from the corresponding author upon reasonable request.

\section{Authors' contributions}

ZD, YZ, HY and ZZ conceived and designed the study. ZD, YZ, HY, GZ, HJ, ZC, YY, ZC, XT, JZ, XL, HX, PL and SG performed the experiments. ZD and ZZ wrote the paper. All authors read and approved the manuscript.

\section{Ethics approval and consent to participate}

All experimental protocols were approved by the Ethics Committee of Zhongshan Hospital, Xiamen University (Xiamen, China) and written informed consent was obtained from all patients. 


\section{Patient consent for publication}

Not applicable.

\section{Competing interests}

The authors declare that they have no competing interests.

\section{References}

1. Heidenreich PA, Albert NM, Allen LA, Bluemke DA, Butler J, Fonarow GC, Ikonomidis JS, Khavjou O, Konstam MA, Maddox TM, et al: Forecasting the impact of heart failure in the United States: A policy statement from the American Heart Association. Circ Heart Fail 6: 606-619, 2013.

2. Marti CN, Georgiopoulou VV and Kalogeropoulos AP: Acute heart failure: Patient characteristics and pathophysiology. Curr Heart Fail Rep 10: 427-433, 2013.

3. Mozaffarian D, Benjamin EJ, Go AS, Arnett DK, Blaha MJ, Cushman M, de Ferranti S, Després JP, Fullerton HJ Howard VJ, et al: Heart disease and stroke statistics-2015 update: A report from the American Heart Association. Circulation 131: e29-e322, 2015.

4. Fabbri A, Marchesini G, Carbone G, Cosentini R, Ferrari A, Chiesa M, Bertini A and Rea F: Acute heart failure in the emergency department: A follow-up study. Intern Emerg Med 11: 115-122, 2016.

5. Collins S, Storrow AB, Albert NM, Butler J, Ezekowitz J, Felker GM, Fermann GJ, Fonarow GC, Givertz MM, Hiestand B, et al: Early management of patients with acute heart failure: State of the art and future directions. A consensus document from the society for academic emergency medicine/heart failure society of America acute heart failure working group. J Card Fail 21: 27-43, 2015.

6. Madika AL, Fertin M, Hebbar E and Lamblin N: 0202: Predictive factors of left ventricular recovery in acute heart failure revealing reduced left ventricular ejection fraction. Arch Cardiovas Dis Suppl 8: 26, 2016.

7. Januzzi JL Jr, Camargo CA, Anwaruddin S, Baggish AL, Chen AA, Krauser DG, Tung R, Cameron R, Nagurney JT, Chae CU, et al: The N-terminal Pro-BNP investigation of dyspnea in the emergency department (PRIDE) study. Am J Cardiol 95: 948-954, 2005 .

8. Roberts E, Ludman AJ, Dworzynski K, Al-Mohammad A, Cowie MR, McMurray JJ and Mant J; NICE Guideline Development Group for Acute Heart Failure: The diagnostic accuracy of the natriuretic peptides in heart failure: Systematic review and diagnostic meta-analysis in the acute care setting. BMJ 350: h910, 2015.

9. Steinhart B, Thorpe KE, Bayoumi AM, Moe G, Januzzi JL Jr and Mazer CD: Improving the diagnosis of acute heart failure using a validated prediction model. J Am Coll Cardiol 54: 1515-1521, 2009.

10. Matsue Y, Suzuki M, Torii S, Yamaguchi S, Fukamizu S, Ono Y, Fujii H, Kitai T, Nishioka T, Sugi K, et al: Prognostic impact of early treatment with tolvaptan in patients with acute heart failure and renal dysfunction. Int J Cardiol 221: 188-193, 2016.

11. Mccullough PA: Scope of cardiovascular complications in patients with kidney disease. Ethn Dis 12: S3-44-8, 2002.

12. Homsak E and Ekart R: Hemodiafiltration affects NT-proBNP but not ST2 serum concentration in end-stage renal disease patients. Clin Biochem 49: 1159-1163, 2016.

13. Wang K, Li HL, Chen LL, Bei WJ, Lin KY, Smyth B, Chen SQ Guo XS, Guo W, Liu YH, et al: Association of N-terminal pro-brain natriuretic peptide with contrast-induced acute kidney injury and long-term mortality in patients with heart failure and mid-range ejection fraction: An observation study. Medicine (Baltimore) 96: e6259, 2017.

14. Chenevier-Gobeaux C, Claessens YE, Voyer S, Ekindjian OG, Ginsburg C and Desmoulins D: Concentrations plasmatiques du peptide natriurétique type $\mathrm{B}(\mathrm{BNP})$ et du fragment $\mathrm{N}$-terminal du propeptide (NT-proBNP) aux urgences: Influence de la fonction rénale. Immuno-Anal Biol Spé 20: 295-300, 2005.

15. Rothenburger M, Stypmann J, Wichter T, et al: The role of NT-proBNP in chronic heart failure and renal insufficiency. Thorac Cardiovasc Surg 53: 618-624, 2005.
16. Herrero-Puente P, Prieto-García B, García-García M, Jacob J, Martín-Sánchez FJ, Pascual-Figal D, Bueno H, Gil V, Llorens P, Vázquez-Alvarez $\mathrm{J}$, et al: Predictive capacity of a multimarker strategy to determine short-term mortality in patients attending a hospital emergency Department for acute heart failure. BIO-EAHFE study. Clin Chim Acta 466: 22-30, 2017.

17. Demissei BG, Cotter G, Prescott MF, Felker GM, Filippatos G, Greenberg BH, Pang PS, Ponikowski P, Severin TM, Wang Y, et al: A multimarker multi-time point-based risk stratification strategy in acute heart failure: Results from the RELAX-AHF trial. Eur J Heart Fail 19: 1001-1010, 2017.

18. Scherer PE, Williams S, Fogliano M, Baldini G and Lodish HF: A novel serum protein similar to Clq, produced exclusively in adipocytes. J Biol Chem 270: 26746-26749, 1995.

19. Pischon T, Girman CJ, Hotamisligil GS, Rifai N, Hu FB and Rimm EB: Plasma adiponectin levels and risk of myocardial infarction in men. JAMA 291: 1730-1737, 2004.

20. Zapata RC, Meachem MD, Cardoso NC, Mehain SO, McMillan CJ, Snead ER and Chelikani PK: Differential circulating concentrations of adipokines, glucagon and adropin in a clinical population of lean, overweight and diabetic cats. BMC Vet Res 13: 85, 2017.

21. Bednarska-Makaruk M, Graban A, Wiśniewska A, Łojkowska W, Bochyńska A, Gugała-Iwaniuk M, Sławińska K, Ługowska A, Ryglewicz D and Wehr H: Association of adiponectin, leptin and resistin with inflammatory markers and obesity in dementia. Biogerontology 18: 561-580, 2017.

22. Mazur-Bialy AI, Bilski J, Wojcik D, Brzozowski B, Surmiak M, Hubalewska-Mazgaj M, Chmura A, Magierowski M, Magierowska K, Mach T and Brzozowski T: Beneficial effect of voluntary exercise on experimental colitis in mice fed a high-fat diet: The role of irisin, adiponectin and proinflammatory biomarkers. Nutrients 9: pii: E410, 2017.

23. Bergmark BA, Cannon CP, White WB, Jarolim P, Liu Y, Bonaca MP, Zannad F and Morrow DA: Baseline Baseline adiponectin concentration and clinical outcomes among patients with diabetes and recent acute coronary syndrome in the EXAMINE trial. Diabetes Obes Metab 19: 962-969, 2017.

24. Ng RC and Chan KH: Potential neuroprotective effects of adiponectin in Alzheimer's disease. Int J Mol Sci 18: pii: E592, 2017.

25. Norvik JV, Schirmer H, Ytrehus K, Jenssen TG, Zykova SN, Eggen AE, Eriksen BO and Solbu MD: Low adiponectin is associated with diastolic dysfunction in women: A cross-sectional study from the Troms $\varnothing$ Study. BMC Cardiovasc Disord 17: 79, 2017.

26. Tsukamoto O, Fujita M, Kato M, Yamazaki S, Asano Y, Ogai A, Okazaki H, Asai M, Nagamachi Y, Maeda N, et al: Natriuretic peptides enhance the production of adiponectin in human adipocytes and in patients with chronic heart failure. J Am Coll Cardiol 53: 2070-2077, 2009.

27. Yanavitski M and Givertz MM: Novel biomarkers in acute heart failure. Curr Heart Fail Rep 8: 206-211, 2011.

28. Yin WH, Wei J, Huang WP, Chen JW, Young MS and Lin SJ: Prognostic value of circulating adipokine levels and expressions of adipokines in the myocardium of patients with chronic heart failure. Circ J 76: 2139-2147, 2012.

29. Yu HP, Jen HL, Yin WH and Wei J: Circulating adiponectin levels following treatment can predict late clinical outcomes in chronic heart failure. Acta Cardiol Sin 33: 139-149, 2017.

30. Park M and Sweeney G: Direct effects of adipokines on the heart: Focus on adiponectin. Heart Fail Rev 18: 631-644, 2013.

31. McMurray JJ, Adamopoulos S, Anker SD, Auricchio A, Böhm M, Dickstein K, Falk V, Filippatos G, Fonseca C, Gomez-Sanchez MA, et al: ESC guidelines for the diagnosis and treatment of acute and chronic heart failure 2012: The task force for the diagnosis and treatment of acute and chronic heart failure 2012 of the European Society of Cardiology. Developed in collaboration with the heart failure association (HFA) of the ESC. Eur J Heart Fail 14: 803-869, 2012.

32. Lamb EJ, Webb MC and O'Riordan SE: Using the modification of diet in renal disease (MDRD) and Cockcroft and Gault equations to estimate glomerular filtration rate (GFR) in older people. Age Ageing 36: 689-692, 2007.

33. Teixeira A, Arrigo M, Tolppanen H, Gayat E, Laribi S, Metra M, Seronde MF, Cohen-Solal A and Mebazaa A: Management of acute heart failure in elderly patients. Arch Cardiovasc Dis 109: 422-430, 2016

34. Fonseca C, Araújo I, Marques F, Brás D and Bettencourt P: A closer look at acute heart failure: Putting Portuguese and European data into perspective. Rev Port Cardiol 35: 291-304, 2016 (In English, Portuguese). 
35. Collins SP, Storrow AB, Levy PD, Albert N, Butler J, Ezekowitz JA, Felker GM, Fermann GJ, Fonarow GC, Givertz MM, et al: Early management of patients with acute heart failure: State of the art and future directions-a consensus document from the SAEM/HFSA acute heart failure working group. Acad Emerg Med 22: 94-112, 2015.

36. Harbaum L, Hennigs JK, Baumann HJ, Lüneburg N, Griesch E, Bokemeyer C, Grünig E and Klose $\mathrm{H}$ : N-terminal pro-brain natriuretic peptide is a useful prognostic marker in patients with pre-capillary pulmonary hypertension and renal insufficiency. PLoS One 9: e94263, 2014.

37. Nishikimi T, Futoo Y, Tamano K, Takahashi M, Suzuki T, Minami J, Honda T, Uetake S, Asakawa H, Kobayashi N, et al: Plasma brain natriuretic peptide levels in chronic hemodialysis patients: Influence of coronary artery disease. Am J Kidney Dis 37: 1201-1208, 2001.

38. McCullough PA: Cardiorenal risk: An important clinical intersection. Rev Cardiovasc Med 3: 71-76, 2002.

39. Bersch-Ferreira ÂC, Sampaio GR, Gehringer MO, Ross-Fernandes MB, Kovacs C, Alves R, Pereira JL, Magnoni CD, Weber B and Rogero MM: Association between polyunsaturated fatty acids and inflammatory markers in patients in secondary prevention of cardiovascular disease. Nutrition 37: 30-36, 2017.

40. Smekal A and Vaclavik J: Adipokines and cardiovascular disease: A comprehensive review. Biomed Pap Med Fac Univ Palacky Olomouc Czech Repub 161: 31-40, 2017.

41. Baldasseroni S, Mannucci E, Orso F, Di Serio C, Pratesi A, Bartoli N, Marella GA, Colombi C, Foschini A, Valoti P, et al: Adiponectin in outpatients with coronary artery disease: Independent predictors and relationship with heart failure. Nutr Metab Cardiovasc Dis 22: 292-299, 2012.

42. Zhang H, Mo X, Hao Y, Huang J, Lu X, Cao J and Gu D: Adiponectin levels and risk of coronary heart disease: A meta-analysis of prospective studies. Am J Med Sci 345: 455-461, 2013.

43. Akbarpour M: The effect of aerobic training on serum adiponectin and leptin levels and inflammatory markers of coronary heart disease in obese men. Biol Sport 30: 21-27, 2013.
44. Ritsinger V, Brismar K, Malmberg K, Mellbin L, Näsman P, Rydén L, Söderberg S, Tenerz Å and Norhammar A: Elevated levels of adipokines predict outcome after acute myocardial infarction: A long-term follow-up of the glucose tolerance in patients with acute myocardial Infarction cohort. Diab Vasc Dis Res 14: 77-87, 2017.

45. Arsenault BJ, Kohli P, Lambert G, DeMicco DA, Laskey R, Messig MM, Kastelein JJ and Waters DD: Emerging cardiovascular disease biomarkers and incident diabetes mellitus risk in statin-treated patients with coronary artery disease (from the treating to new targets [TNT] study). Am J Cardiol 118: 494-498, 2016.

46. Dries DL, Exner DV, Domanski MJ, Greenberg B and Stevenson LW: The prognostic implications of renal insufficiency in asymptomatic and symptomatic patients with left ventricular systolic dysfunction. J Am Coll Cardiol 35: 681-689, 2000.

47. Lim CC, Teo BW, Tai ES, Lim SC, Chan CM, Sethi S, Wong TY and Sabanayagam C: Elevated serum leptin, adiponectin and leptin to adiponectin ratio is associated with chronic kidney disease in Asian adults. PLoS One 10: e0122009, 2015.

48. Markaki A, Psylinakis E and Spyridaki A: Adiponectin and end-stage renal disease. Hormones (Athens) 15: 345-354, 2016.

49. Taherimahmoudi M, Ahmadi H, Mehrsai A and Pourmand G: Plasma adiponectin concentration and insulin resistance: Role of successful kidney transplantation. Transplant Proc 42: 797-800, 2010.

50. Bakkaloglu SA, Buyan N, Funahashi T, Pasaoglu H, Elhan AH, Hasanoglu E and Soylemezoglu O: Adiponectin levels and atherosclerotic risk factors in pediatric chronic peritoneal dialysis patients. Perit Dial Int 25: 357-361, 2005.

51. Kamimura MA, Canziani ME, Sanches FR, Velludo CM, Carrero JJ, Bazanelli AP, Draibe SA and Cuppari L: Variations in adiponectin levels in patients with chronic kidney disease: A prospective study of 12 months. J Bras Nefrol 34: 259-265, 2012. 